\title{
Determination of the chemical components and phospholipids of velvet antler using UPLC/QTOF-MS coupled with UNIFI software
}

\author{
LI-QUN ZHANG ${ }^{1}$, JIA WANG ${ }^{2}$, TING LI ${ }^{2}$, PING-YA LI ${ }^{2}$, YUN-HUA WANG ${ }^{2}$, \\ MIAO YANG ${ }^{2}$, JIN-PING LIU ${ }^{2}$ and JI-HUA LIU ${ }^{2}$ \\ ${ }^{1}$ Department of Obstetrics and Gynecology, The First Hospital of Jilin University; \\ ${ }^{2}$ Department of Natural Medicinal Chemistry, School of Pharmaceutical Sciences, \\ Jilin University, Changchun, Jilin 130021, P.R. China
}

Received June 8, 2018; Accepted November 11, 2018

DOI: $10.3892 /$ etm.2019.7372

\begin{abstract}
Velvet antler, which exhibits immune and growth enhancing effects, is commonly used in a variety of Asian health care products, but its complex components remain unknown. The current study analyzed extracts using ultra-performance liquid chromatography coupled with electrospray ionization quadrupole time-of-flight tandem mass spectrometry in the $\mathrm{MS}^{\mathrm{E}}$ mode. Automated detection and data filtering were performed using UNIFI software and peaks were compared with a proprietary scientific library (Traditional Medicine Library; TML). The results obtained using different data processing parameters (including 3D peak detection, target by mass and fragment identification) were evaluated against 87 compounds comprising 1 lignan, 30 terpenoids (including 20 triterpenes), 39 steroids, 8 alkaloids, 4 organic acids and 5 esters in the TML. Using a screening method with a mass accuracy cutoff of $\pm 2 \mathrm{mDa}$, a retention time cutoff of $\pm 0.2 \mathrm{~min}$, a minimum response threshold of 1,000 counts and an average of 10 false detects per sample analysis, 16 phospholipids were identified in the extracts of velvet antler, three of which were quantified. The results demonstrated that there was $1.07 \pm 0.02 \mu \mathrm{g} / \mathrm{g}$ of 1-myristoyl-sn-glycero-3-phosphocholine, $7.05 \pm 0.52 \mathrm{ng} / \mathrm{g}$ of 1,2-dimyristoyl-sn-glycero-3-phosphocholine and $18.81 \pm 0.55 \mathrm{ng} / \mathrm{g}$ of 1-palmitoyl-2-oleoyl-sn-glycero-3-phos-
\end{abstract}

Correspondence to: Professor Ji-Hua Liu, Department of Natural Medicinal Chemistry, School of Pharmaceutical Sciences, Jilin University, 1266 Fujin Road, Changchun, Jilin 130021, P.R. China E-mail: liujh@jlu.edu.cn

Abbreviations: UPLC/QTOF-MS, ultra-performance liquid chromatography- quadrupole-time-of-flight mass spectrometry; MPC, 1-myristoyl-sn-glycero-3-phosphocholine; DPC, dimyristoylsn-glycero-3-phosphocholine; POPC, 1-palmitoyl- 2-oleoyl-snglycero-3-phosphocholine; EVA, extract of velvet antler; TML, Traditional Medicine Library; GPLs, glycerophospholipids.

Key words: antler velvet, phospholipids, ultra-performance liquid chromatography-quadrupole-time-of-flight mass spectrometry, component identification phocholine in velvet antler. The current study successfully identified certain components of velvet antler. Furthermore, the results may provide an experimental basis for further pharmacological and clinical study.

\section{Introduction}

Velvet antler growth is a rapid process (1). During this period, constitutive tissues including bone, cartilage, skin, nerves and blood vessels grow quickly (2). Previous studies have demonstrated that certain growth factors, including vascular endothelial growth factor, epidermal growth factor, fibroblast growth factor and nerve growth factor are abundant in velvet antler and are responsible for rapid tissue growth (3-6). Numerous studies have also revealed that velvet antler contains amino acids (7-9), polypeptides $(10,11)$, proteins $(9,12-15)$ and phospholipids (16). However, there have been few reports that assess the phytochemical components of velvet antler, which are highly biologically active.

Ultra-performance liquid chromatography coupled with electrospray ionization quadrupole time-of-flight tandem mass spectrometry (UPLC/ESI-QTOF-MS) has been utilized to reliably analyze the complex composition of food, herbal medicines and biological samples (17-20). QTOF-MS analyzers offer a high mass resolution, sensitivity and accuracy, providing accurate ion masses to determine molecular formulas (17-20). The present study developed a method of UPLC/QTOF-MS on a Waters Xevo G2-XS QTOF system for the determination of the chemical components of velvet antler. Based on accurate mass measurements, a total of 87 compounds were detected in the velvet antler and tentatively identified using the Traditional Medicine Library (TML) of the UNIFI platform.

Phospholipids are major constituents of biological membranes, which maintain membrane integrity and cell homeostasis $(21,22)$. Glycerophospholipids (GPLs) are the largest family of amphiphilic phospholipids (23). They are comprised of a glycerol backbone containing fatty acid chains that are acylated at the sn- 1 and sn-2 position and possess a polar head group containing a phosphate esterified at the sn-3 position, which is attached to a polyol or amino acid moiety (23). The following classes of GPLs are defined by the structure of the head group: phosphatidylserine, phosphatidylglycerol, 
phosphatidylethanolamine, phosphatidylinositol, phosphatidic acid and phosphatidylcholine (23). Each class of GLP comprises many species that possess the same head group but different chain lengths, number of unsaturations and substitution positions of the esterified fatty acid (23). Various methods have been utilized for the quantification and structural determination of individual molecular species of phospholipids. For example, thin-layer chromatography was used in the chemical analysis of components from velvet antlers (24). Gas chromatography techniques are not so generalized for determining the components from velvet antler, due to analytical difficulty, even following derivatization, and the long time required for these reactions and the low limits of detection (LOD) (25). QTOF-MS has several advantages, including a high reliability, short analysis time, a small required sample quantity and a high accuracy in the identification and quantification of phospholipids $(26,27)$.

The present study determined the phospholipids present in velvet antler, which was determined via qualitative-quantitative analysis using the UPLC/QTOF-MS method. Previously, Zhou and Li (26) identified 5 phospholipids in the velvet antlers of sika deer including sphingomyelin, phosphatidylcholine, phosphatidylethanolamine, lysophosphatidylcholine and lysophosphatidylethanolamine. The present study identified 16 phospholipids from velvet antler extracts, with 3 phospholipids being quantified. Consequently, a UPLC/QTOF-MS method with commendable validation results, a slope of the standard curve and precision was developed and validated for the quantitative detection of phospholipids and the identification of complex components in velvet antler. This method may provide an experimental basis for further pharmacological and clinical applications of velvet antler products.

\section{Materials and methods}

Materials. Antler velvet (Cervus nippon Temminck var. mantchurieus Sainhoe) was collected from farmed sika deer in the Shuangyan district of Changchun (Jilin Ruikang Biotechnology Co., Ltd., Liaoyuan, China). The upper section was cut to a thickness of 2-3 mm, freeze dried and then powdered to $160-180$ mesh $(84-95 \mu \mathrm{m})$. Antler velvet samples were identified at School of Pharmaceutical Sciences in Jilin University (Changchun, China).

Mass spectrometry grade acetonitrile and methanol were purchased from Thermo Fisher Scientific, Inc., (Waltham, MA, USA). Phospholipid standards (1-myristoyl-sn-glycero-3-phosphocholine, 98.5\%; dimyristoyl-sn-glycero-3-phospho choline, 98.5\%; and 1-palmitoyl-2-oleoyl-sn-glycero-3-phosphocholine, $98.5 \%$ ) were purchased from Nanjing NutriHerb BioTech Co., Ltd., (Jiangsu, China). Formic acid was purchased from Sigma-Aldrich (Merck KGaA, Darmstadt, Germany). Leucine-enkephalin (99\%) was also purchased from Sigma-Aldrich; Merck KGaA. Deionized water was prepared using a Milli-Q system (EMD Millipore, Billerica, MA, USA). All other reagents were of analytical grade.

UPLC/QTOF-MS conditions. An ACQUITY UPLC I-Class System coupled to a Waters Xevo G2-XS QTOF mass spectrometer detector (Waters UK, Elstree, UK) was used. All chromatographic and MS equipment was purchased from
Waters Corporation (Milford, MA, USA). Chromatographic separations were achieved using an ACQUITY UPLC ${ }^{\circledR}$ BEH C18, $1.7 \mu \mathrm{m}(2.1 \times 50 \mathrm{~mm}$; Waters Corporation) capillary column. Analytical column chromatography was performed at $40^{\circ} \mathrm{C}$. The mobile phase consisted of a mixture of acetonitrile, water and formic acid at a flow rate of $0.4 \mathrm{ml} \mathrm{min} \mathrm{min}^{-1}$. Acetonitrile with $0.1 \%$ formic acid was used as mobile phase $\mathrm{A}$ and water with $0.1 \%$ formic acid was used as mobile phase B. A 90/10 mixture of water/acetonitrile was utilized as the weak wash solvent and 50/50 water/acetonitrile was used as the strong wash solvent for rinsing the injection needle. Prior to running the elution, the column was equilibrated to $35 \%$. The elution gradient program was $35-82 \%$ A from 0-3 min, $82 \%$ A from $3-7 \mathrm{~min}, 35-82 \% \mathrm{~A}$ from $7-8 \mathrm{~min}$ and $35 \% \mathrm{~A}$ from 8-9 $\mathrm{min}$.

MS experiments were performed using a Waters Xevo G2-XS QTOF mass spectrometer connected to the ACQUITY UPLC I-Class System via an electrospray ionization (ESI) interface. Atmospheric pressure ionization was performed in positive ion, negative ion and sensitivity analyzer modes for QTOF-MS data acquisition. A wide mass range (m/z 100-1200) was selected for the acquisition of accurate mass precursor and fragment ion data. The corona voltage, sampling cone voltage, source temperature and desolvation temperature was $3.0 \mathrm{kV}$, $40 \mathrm{~V}, 100^{\circ} \mathrm{C}$ and $350^{\circ} \mathrm{C}$, respectively. Nitrogen $\left(20 \pm 2^{\circ} \mathrm{C} ; 10 \mathrm{psi}\right)$ was used for desolvation and the cone gas flow rate was 800 and $50 \mathrm{l} \mathrm{h}^{-1}$, respectively. Argon was used as the collision gas and the collision energy was $15-45 \mathrm{~V}$ for high energy ionizations. Data were acquired and analyzed using MassLyn ${ }^{\mathrm{TM}} \mathrm{NT}$ 4.1 software (Waters Corporation). Analyses were performed in full scan mode and the scan time was set to $0.2 \mathrm{sec}$. To ensure for mass accuracy and reproducibility of the optimized MS conditions, leucine-enkephalin (m/z 554.2615 in negative mode and m/z 556.2771 in positive mode) was used as a reference (lock mass) at a concentration of $200 \mathrm{pg} / \mathrm{ml}$ and a flow rate of $10 \mu \mathrm{l} / \mathrm{min}$. The reference was injected into the MS instrument every $10 \mathrm{sec}$. The instrument was calibrated using sodium formate solution as the calibration standard to achieve mass accuracies of $<0.5 \mathrm{mDa}$.

Accurate mass screening of the constituents of velvet antler. The UNIFI 1.8 informatics platform (Waters Corporation) was utilized to integrate data acquisition, data mining, library searching and to generate a report $(27,28)$. The raw data were imported and screened against the TML and a customized phospholipid library produced in the current study. A natural product analytical workflow within UNIFI was used to analyze the chromatographic and mass spectral data of the velvet antler extract components utilizing various in-built tools, including the customized library and filters.

The TML of the UNIFI software contains 6,415 compounds and their associated data. A customized library was also created in the current study that comprised 45 phospholipids with detailed metadata (including the molecular structure and compound name) based on their chemical structure. Compound screening was performed by setting a mass tolerance of $2 \mathrm{mDa}$, a retention time cutoff of $\pm 0.2 \mathrm{~min}$, counts $>1,000$ and a minimum of 5 fragmentations, and a mean of 10 false detects per sample analysis. To demonstrate the validity of these results three standards were 


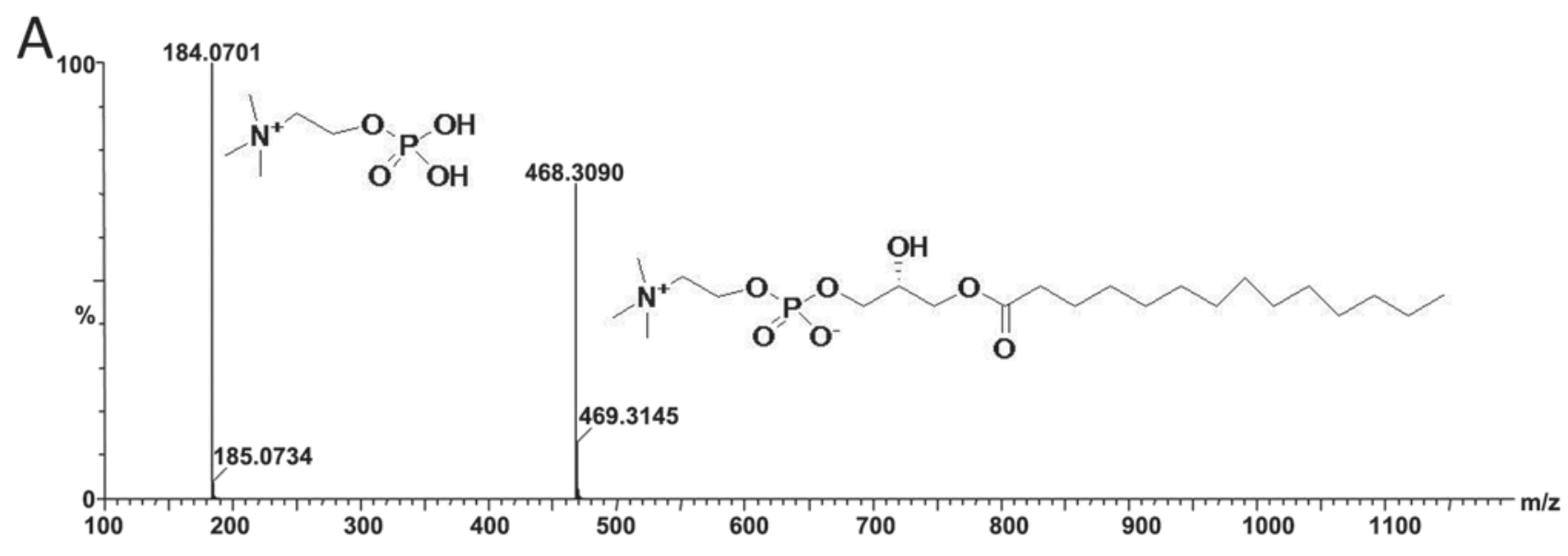

B
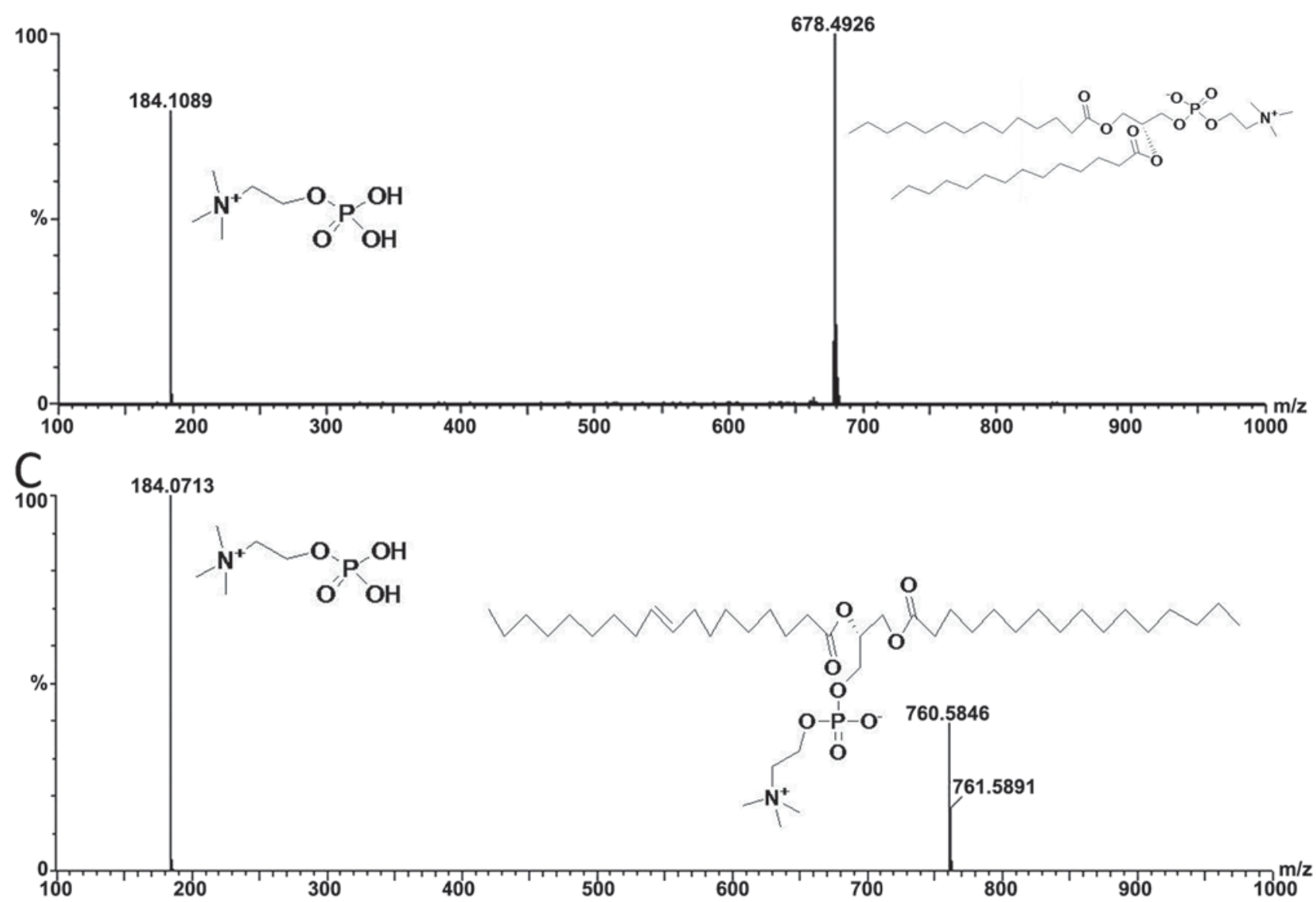

Figure 1. Chemical structures and daughter scan mode (tandem mass spectrometry) spectra of (A) MPC, (B) DPC and (C) POPC. MPC, 1-myristoyl-sn-glycero-3phosphocholine; EVA, extract of antler velvet; DPC, 1,2-dimyristoyl-sn-glycero-3-phosphocholine; POPC, 1-palmitoyl- 2-oleoyl-sn-glycero-3-phosphocholine.

utilized: 1-myristoyl-sn-glycero-3-phosphocholine (MPC), 1,2-dimyristoyl-sn-glycero-3-phosphocholine (DPC) and 1-palmitoyl- 2-oleoyl-sn-glycero-3-phosphocholine (POPC). Their retention times and accurate masses were compared with the sample of velvet antler extract.

Extraction of velvet antler. An ultrasound-assisted extraction $\left(25^{\circ} \mathrm{C} ; 40 \mathrm{kHz}\right)$ of $100 \mathrm{~g}$ velvet antler powder was performed with $3 \times 150 \mathrm{ml}$ ethanol for $10 \mathrm{~min}$ each time. The solid was filtered in each step and the pore size was 30-50 $\mu \mathrm{m}$. The filtered and pooled liquid phases were concentrated to $3 \mathrm{ml}$ under a reduced pressure at $-20^{\circ} \mathrm{C}$. Subsequently, $6 \mathrm{ml}$ acetone was added to generate a white precipitate. Then the mixture was centrifuged at $2,012 \mathrm{x} \mathrm{g}$ at $20^{\circ} \mathrm{C}$ for $10 \mathrm{~min}$, the supernatant was removed, and the precipitate was stored at $-20^{\circ} \mathrm{C}$ until analysis. The precipitate was dissolved in methanol to achieve the concentration of $40 \mathrm{mg} / \mathrm{ml}$, and $2 \mathrm{ml}$ solution was filtered by $0.22-\mu \mathrm{m}$ microporous filter membrane and put into 


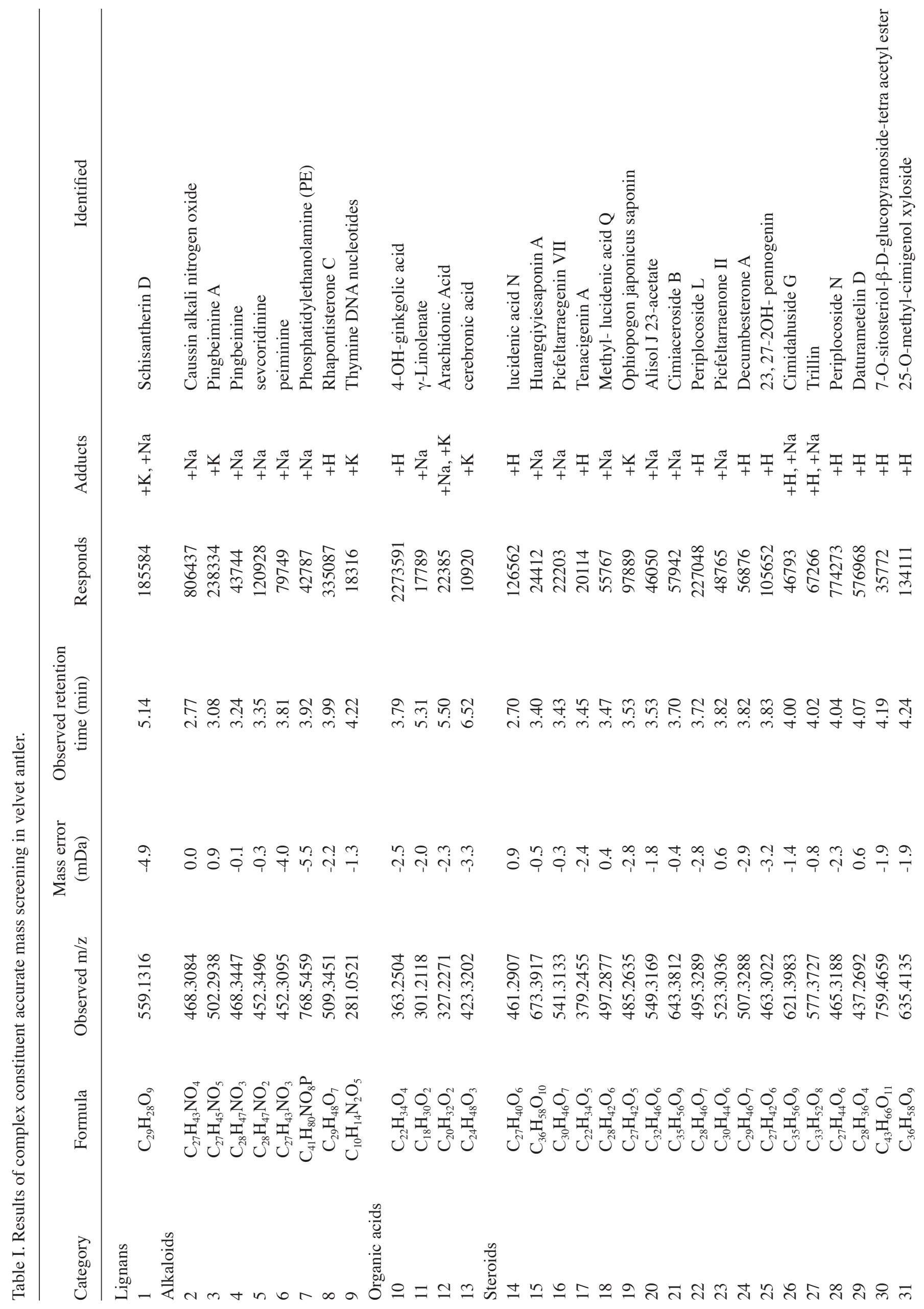




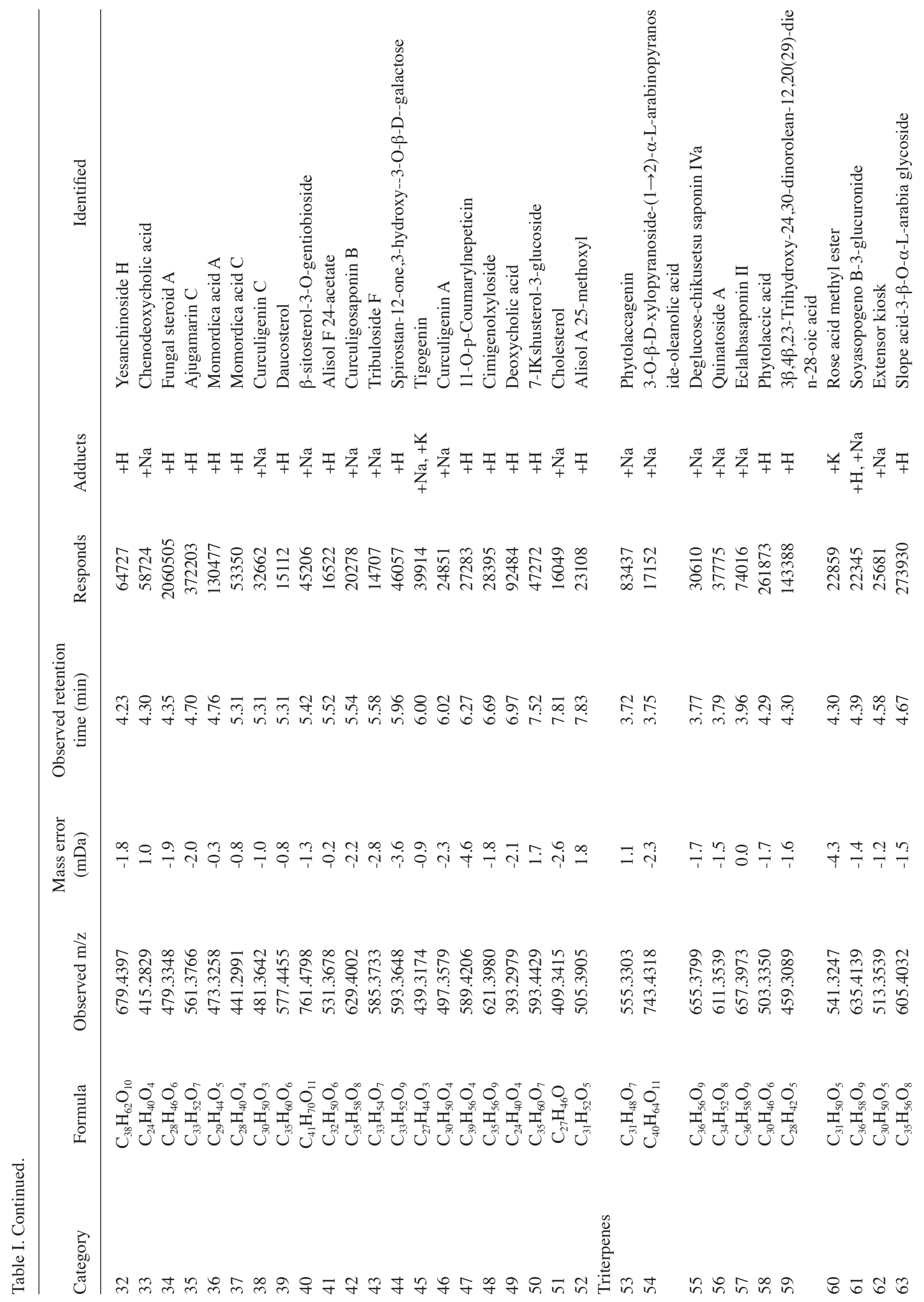




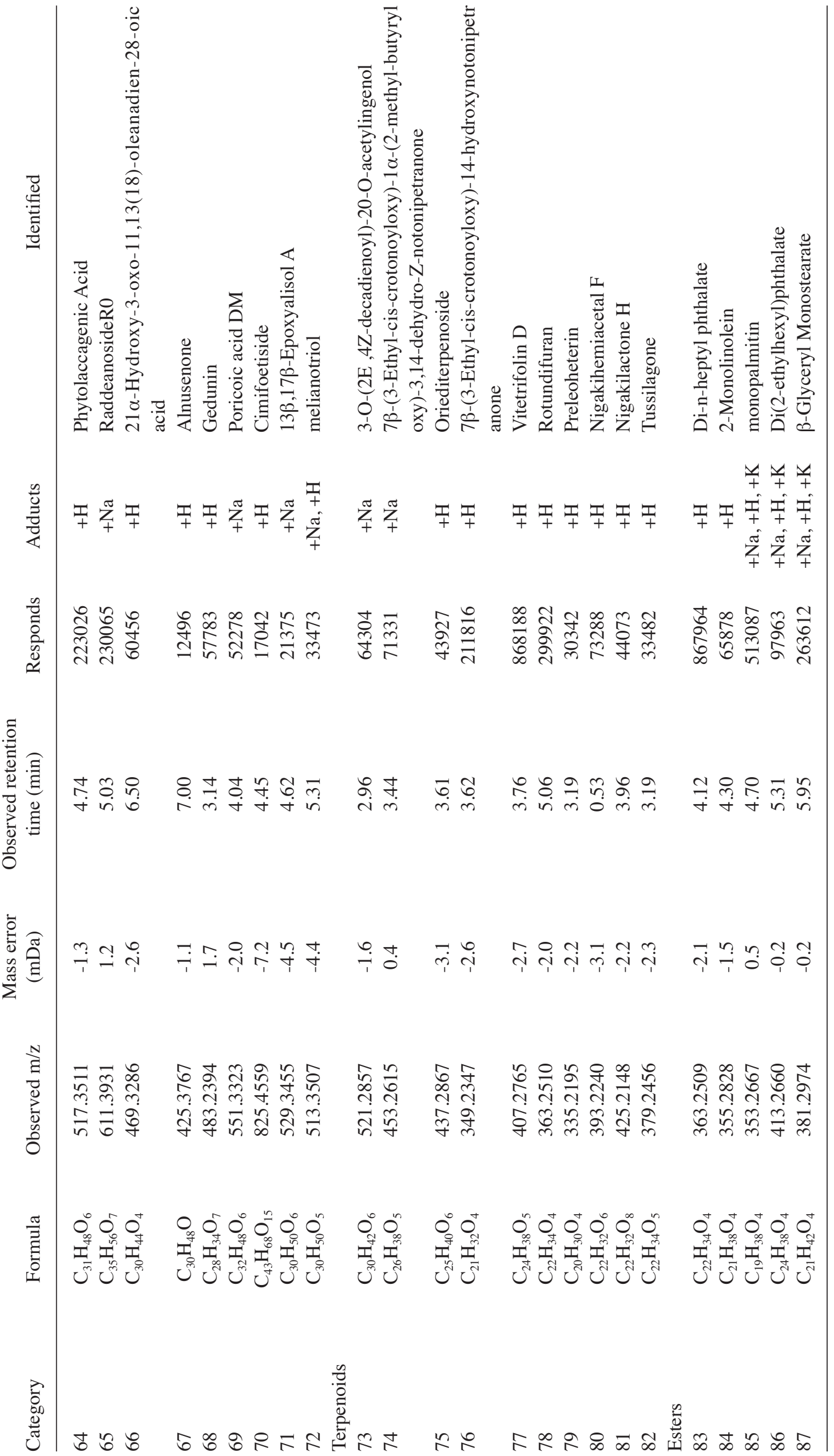




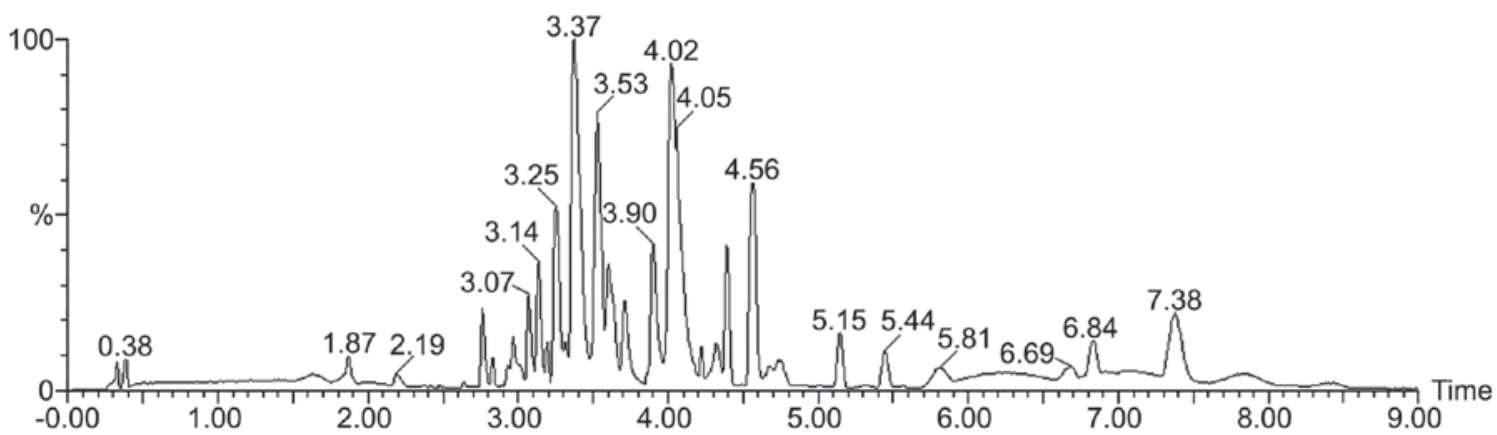

Figure 2. Ultra-performance liquid chromatography coupled with electrospray ionization quadrupole time-of-flight tandem mass spectrometry BPI chromatogram of velvet antler extract.

automatic sampling bottle prior injection of $5 \mu 1$ methanolic solution into the UPLC system. Internal standards with were added to the antler powder to allow commenting on the extraction procedure. The mean recovery rates were in the range of $90-110 \%$.

Qualitative determination of $M P C, D P C$ and $P O P C$ in EVA samples by UPLC/QTOF-MS/MS. The MS data of the 3 phospholipid standards were initially assessed using either ESI or the atmospheric pressure chemical ionization (APCI) mode. ESI was selected as the ionization mode for the present experiments as it provides greater analyte responses than those achieved with APCI. Furthermore, high ionization efficiency was observed under ESI conditions when monitoring the signal in positive ion mode. Following instrument parameter optimization to achieve the highest sensitivity and lowest background noise for the protonated molecules of MPC, DPC and POPC, the ion transition $(\mathrm{m} / \mathrm{z})$ $468.30 \rightarrow 184.07$ was selected for the quantification of MPC, $\mathrm{m} / \mathrm{z} 678.49 \rightarrow 184.10$ for DPC and $\mathrm{m} / \mathrm{z} 760.58 \rightarrow 184.07$ for POPC (Fig. 1). Other UPLC and MS conditions were the same as those aforementioned.

Method validation of phospholipid quantitative detection. The method of quantitative phospholipid detection was fully validated according to the guidelines set by the US Food and Drug Administration. Specificity was tested by inspecting the solvent used in each validation run for interfering peaks. The calibration curve was determined by plotting the peak area vs. the corresponding concentration of injected standards. The limit of quantitation (LOQ) was the concentration that exhibited an identifiable and reproducible analyte peak (response) with a precision of $10 \%$ and an accuracy of $90-110 \%$. Additionally, the analyte response at the LOQ should be at least ten times the response of the blank sample. Intra- and inter-day precision and accuracy were determined from 6 replicates of the QC samples analyzed on the same day and on 3 different days. At each concentration, acceptable precision (repeatability) and accuracy were defined as the relative standard deviation (RSD) of $<10 \%$ and a relative error within $\pm 10 \%$. The sample recovery was calculated at three different concentrations by comparing the peak areas of the sample and the peaks in samples spiked with standard. Short- and long-term stability were investigated by reanalyzing the quality control batches following storage at $-20^{\circ} \mathrm{C}$ for 30 days and at room temperature for $12 \mathrm{~h}$, respectively.

\section{Results}

Characterization of velvet antler complex constituents. A total of 87 compounds in velvet antler were identified or tentatively characterized. These included: 1 lignan, 30 terpenoids (including 20 triterpenes), 39 steroids, 8 alkaloids, 4 organic acids and 5 esters (Table I; Fig. 2). The compounds were identified based on accurate mass measurements, tandem MS behaviors, database matching and comparison to reference standards, considering all data reported in the literature. The advantages of using UPLC for the analysis of samples with complex components (including enhance separation efficiency and higher peak capacity) are fully demonstrated here, for example via the shorter chromatographic peaks (Fig. 2). Enhanced separation efficiency (sharper chromatographic peaks) and higher peak capacity were observed in the analysis at $10 \mathrm{~min}$. The raw data includes the molecular weight of the compounds and the respective fragment ion information, which may be matched to the TML for in-depth ingredient analysis and structural identification (Data not shown).

Velvet antler phospholipid identification. A customized library was constructed to verify the identity of velvet antler phospholipids on the basis of previously reported data (16,21-24). A total of 45 phospholipids were added to the custom library with details including compound name, chemical structure and chemical formula. As a result, 16 phospholipids were identified or tentatively characterized from the velvet antler extract. These data, including retention time, formula, mass error, adducts and compound names are presented in Table II. The use of mass spectrometry-based approaches alone is insufficient for the identification of complex botanical chemical components (27). Therefore, the present study utilized reference standards to validate these compounds, enhancing the accuracy and reliability of the results obtained. The standards of MPC, DPC and POPC were assayed under optimized conditions and their spectra and chromatograms were compared with those of the EVA samples. The results confirmed that MPC, DPC and POPC were present in the EVA. The chromatograms and spectra are presented in Figs. 1 and 3. 
Table II. Identification of velvet antler phospholipids.

\begin{tabular}{|c|c|c|c|c|c|c|}
\hline No. & Formula & $\begin{array}{l}\text { Observed } \\
\mathrm{m} / \mathrm{z}\end{array}$ & $\begin{array}{l}\text { Mass } \\
\text { error } \\
(\mathrm{mDa})\end{array}$ & $\begin{array}{l}\text { Observed } \\
\text { retention } \\
\text { time }(\min )\end{array}$ & Adducts & Identified \\
\hline 1 & $\mathrm{C}_{45} \mathrm{H}_{87} \mathrm{O}_{13} \mathrm{P}$ & 905.5428 & -8.8 & 2.30 & $+\mathrm{K}$ & L-alpha-Phosphatidylinositol-4,5-bisphosphate \\
\hline 2 & $\mathrm{C}_{22} \mathrm{H}_{46} \mathrm{NO}_{7} \mathrm{P}$ & 468.3084 & -0.1 & 2.77 & $+\mathrm{H}$ & 1-Myristoyl-2-hydroxy-sn-glycero-3-phosphocholine \\
\hline 3 & $\mathrm{C}_{21} \mathrm{H}_{40} \mathrm{NaO}_{7} \mathrm{P}$ & 459.2481 & -0.1 & 3.38 & $+\mathrm{H}$ & $\begin{array}{l}\text { 1-Oleoyl-sn-glycero-3-lysophosphatidic acid sodium } \\
\text { salt }\end{array}$ \\
\hline 4 & $\mathrm{C}_{26} \mathrm{H}_{54} \mathrm{NO}_{7} \mathrm{P}$ & 524.3714 & 0.3 & 4.03 & $+\mathrm{H}$ & $\beta$-Acetyl- $\gamma$-O-hexadecyl-L- $\alpha$-phosphatidylcholine \\
\hline 5 & $\mathrm{C}_{42} \mathrm{H}_{78} \mathrm{NaO}_{10} \mathrm{P}$ & 835.4824 & -3.8 & 4.18 & $+\mathrm{K}$ & $\begin{array}{l}\text { 1,2-Dioleoyl-sn-glycero-3-phospho-rac-(1-glycerol) } \\
\text { sodium salt }\end{array}$ \\
\hline 6 & $\mathrm{C}_{42} \mathrm{H}_{82} \mathrm{NO}_{10} \mathrm{P}$ & 814.5507 & -6.1 & 4.19 & $+\mathrm{Na}$ & Phosphatidylserine \\
\hline 7 & $\mathrm{C}_{33} \mathrm{H}_{66} \mathrm{NO}_{8} \mathrm{P}$ & 674.4082 & -7.5 & 4.22 & $+\mathrm{K}$ & 1,2-Dimyristoyl-sn-glycero-3-phosphoethanolamine \\
\hline 8 & $\mathrm{C}_{42} \mathrm{H}_{81} \mathrm{Na}_{2} \mathrm{O}_{10} \mathrm{P}$ & 861.5002 & 0.8 & 4.32 & $+\mathrm{K}$ & $\begin{array}{l}\text { 1,2-Distearoyl-sn-glycero-3-phospho-rac-glycerol } \\
\text { sodium salt }\end{array}$ \\
\hline 9 & $\mathrm{C}_{29} \mathrm{H}_{58} \mathrm{NO}_{8} \mathrm{P}$ & 580.3977 & 0.4 & 4.34 & $+\mathrm{H}$ & 1,2-Dilauroyl-sn-glycero-3-phosphoethanolamine \\
\hline 10 & $\mathrm{C}_{42} \mathrm{H}_{82} \mathrm{NO}_{8} \mathrm{P}$ & 760.5794 & -5.7 & 4.58 & $+\mathrm{H}$ & 2-Oleoyl-1-palmitoyl-sn-glycero-3-phosphocholine \\
\hline 11 & $\mathrm{C}_{36} \mathrm{H}_{72} \mathrm{NO}_{8} \mathrm{P}$ & 716.4543 & -8.4 & 4.60 & $+\mathrm{K}$ & 1,2-Dimyristoyl-sn-glycero-3-phosphocholine \\
\hline 12 & $\mathrm{C}_{42} \mathrm{H}_{80} \mathrm{NO}_{8} \mathrm{P}$ & 780.5484 & -2.9 & 5.26 & $+\mathrm{Na}$ & L-A-phosphatidylcholine \\
\hline 13 & $\mathrm{C}_{39} \mathrm{H}_{69} \mathrm{O}_{8} \mathrm{P}$ & 697.4815 & 1.2 & 5.37 & $+\mathrm{H}$ & L- $\alpha$-phosphatidic acid \\
\hline 14 & $\mathrm{C}_{35} \mathrm{H}_{67} \mathrm{Na}_{2} \mathrm{O}_{8} \mathrm{P}$ & 693.4433 & -0.9 & 5.49 & $+\mathrm{H}$ & $\begin{array}{l}\text { L- } \beta, \gamma \text {-Dipalmitoyl-L- } \alpha \text {-phosphatidicaciddisodium } \\
\text { salt }\end{array}$ \\
\hline 15 & $\mathrm{C}_{44} \mathrm{H}_{88} \mathrm{NO}_{8} \mathrm{P}$ & 790.6295 & -2.5 & 6.02 & $+\mathrm{H}$ & 1,2-Distearoyl-rac-glycero-3-phosphocholine \\
\hline 16 & $\mathrm{C}_{40} \mathrm{H}_{80} \mathrm{NO}_{8} \mathrm{P}$ & 772.5217 & -3.7 & 7.01 & $+\mathrm{K}$ & 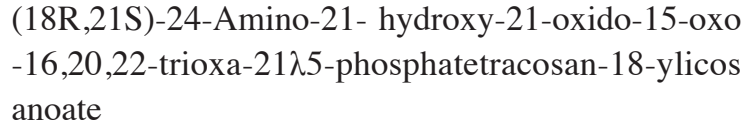 \\
\hline
\end{tabular}
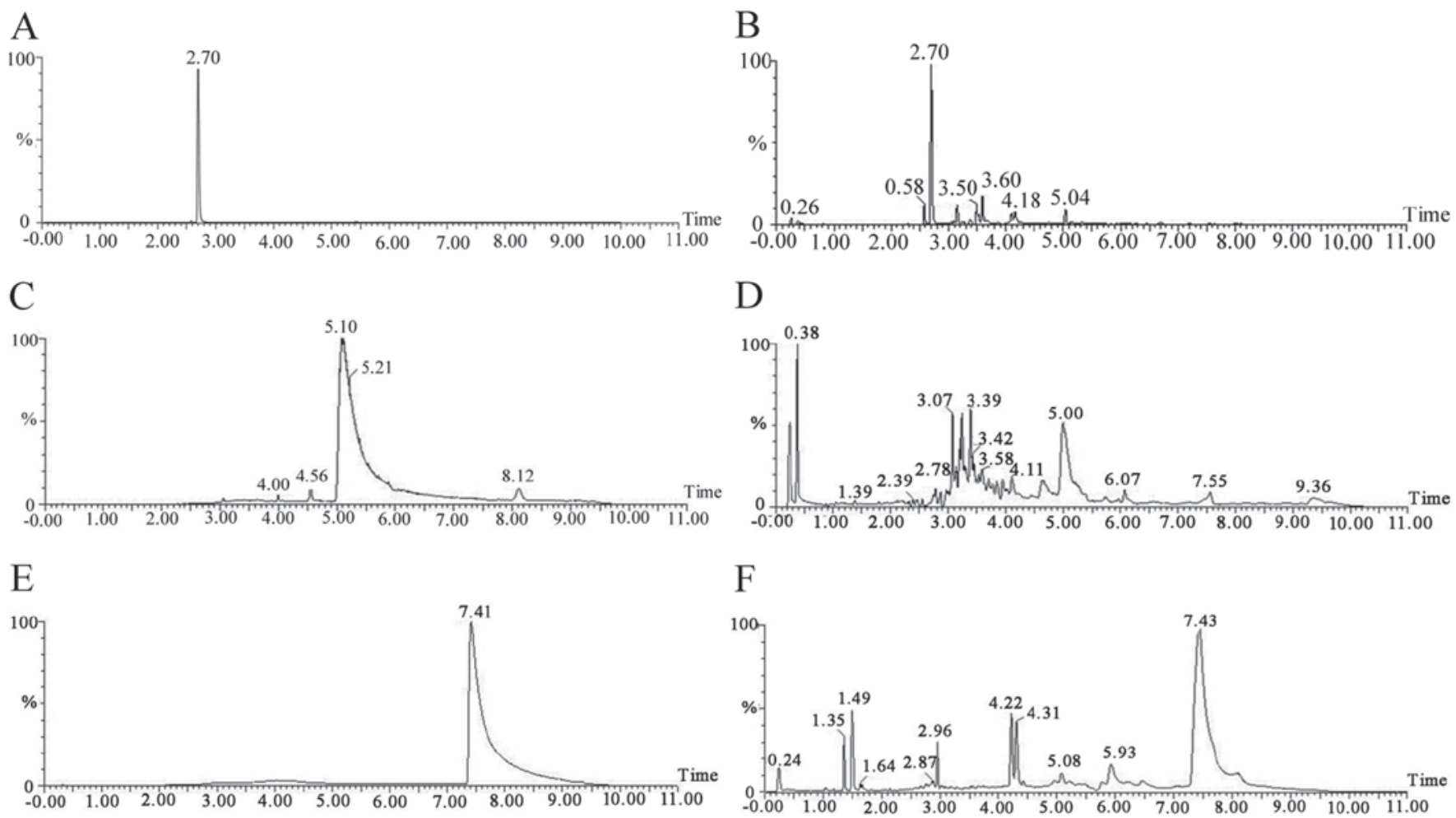

Figure 3. Tandem mass spectrometry chromatograms of the phospholipid standards and velvet antler extract. The results for (A) MPC standard, (B) MPC in EVA, (C) DPC standard, (D) DPC in EVA, (E) POPC standard and (F) POPC in EVA are presented. MPC, 1-myristoyl-sn-glycero-3-phosphocholine; EVA, extract of antler velvet; DPC, 1,2-dimyristoyl-sn-glycero-3-phosphocholine; POPC, 1-palmitoyl- 2-oleoyl-sn-glycero-3-phosphocholine. 
Table III. Quantitative detection of MPC, DPC and POPC in the extract of velvet antler.

\begin{tabular}{lccc}
\hline Phospholipids & Calibration curve & $\mathrm{r}^{2}$ & Content of phospholipids in velvet antler \\
\hline MPC & $\mathrm{Y}=7362.7 \mathrm{X}+21137.8$ & 0.9996 & $1.07 \pm 0.02 \mu \mathrm{g} / \mathrm{g}$ \\
DPC & $\mathrm{Y}=39811.5 \mathrm{X}+78542.2$ & 0.9992 & $7.05 \pm 0.52 \mathrm{ng} / \mathrm{g}$ \\
POPC & $\mathrm{Y}=1653604.2 \mathrm{X}-53602.4$ & 0.9998 & $18.81 \pm 0.55 \mathrm{ng} / \mathrm{g}$ \\
\hline
\end{tabular}

MPC, 1-myristoyl-sn-glycero-3-phosphocholine; DPC, 1,2-dimyristoyl-sn-glycero-3-phosphocholine; POPC, 1-palmitoyl-2-oleoyl-sn-glycero3-phosphocholine.

Table IV. Intra- and inter-day accuracy and precision of quality control samples.

\begin{tabular}{|c|c|c|c|c|c|}
\hline \multirow[b]{2}{*}{ Samples } & \multirow[b]{2}{*}{ Concentration $(\mu \mathrm{g} / \mathrm{ml})$} & \multicolumn{2}{|c|}{ Inter-day $(n=6)$} & \multicolumn{2}{|c|}{ Intra-day (n=3) } \\
\hline & & Precision (RSD\%) & Accuracy (RE\%) & Precision (RSD\%) & Accuracy (RE\%) \\
\hline \multirow[t]{3}{*}{ MPC } & 10 & 4.1 & -2.1 & 5.9 & 3.2 \\
\hline & 20 & 3.5 & -3.6 & 4.5 & 2.5 \\
\hline & 30 & 2.8 & -1.8 & 4.1 & -2.9 \\
\hline \multirow[t]{3}{*}{ DPC } & 5 & 1.8 & 2.5 & 3.9 & -2.3 \\
\hline & 10 & 1.6 & -2.0 & 3.7 & -4.0 \\
\hline & 20 & 1.2 & -1.8 & 5.6 & -3.3 \\
\hline \multirow{3}{*}{ POPC } & 15 & 3.4 & -2.7 & 6.5 & -4.5 \\
\hline & 20 & 3.6 & 3.2 & 5.5 & -2.4 \\
\hline & 30 & 2.7 & -3.3 & 5.1 & -2.8 \\
\hline
\end{tabular}

RSD, relative standard deviation; RE, relative error; MPC, 1-myristoyl-sn-glycero-3-phosphocholine; DPC, 1,2-dimyristoyl-sn-glycero-3-phosphocholine; POPC, 1-palmitoyl- 2-oleoyl-sn-glycero-3-phosphocholine.

Quantitative analysis of MPC, DPC and POPC in EVA. The linearity, LODs, LOQs, precision, repeatability, stability and recovery of MPC, DPC and POPC were determined using the optimized UPLC/QTOF-MS/MS method. The calibration curves of each are presented in Table III. The results demonstrated that the correlation coefficients were all $>0.9995$, indicating that good linear correlations were achieved. The RSDs of the intra-day and inter-day precisions were deemed to be acceptable (Table IV). The results of the repeatability and stability tests, and the mean recovery rates were also deemed to be in the range of $90-110 \%$, indicating that the qualitative method was accurate, reproducible and reliable for the assessment of MPC, DPC and POPC in the EVA.

The aforementioned UPLC/QTOF-MS/MS analytical method was subsequently used to quantify the three phospholipids present in the EVA samples. Each standard was analyzed in triplicate and each sample was analyzed once to determine the average content of the constituents. The analytical results are presented in Table III. The results demonstrated that there was $1.07 \pm 0.02 \mu \mathrm{g} / \mathrm{g}$ of MPC, $7.05 \pm 0.51 \mathrm{ng} / \mathrm{g}$ of DPC and $18.81 \pm 0.55 \mathrm{ng} / \mathrm{g}$ of POPC in the EVA.

\section{Discussion}

The chemical composition of velvet antler was determined in the present study using the UPLC/QTOF/MS method combined with UNIFI software for component screening.
Compared with traditional identification methods that require long and complex purification procedures and structure identification by nuclear magnetic resonance (NMR) and mass spectrometry, the method used in the present study is simple, fast and easy to operate. Although the results of component screening are based on the mass ratio of parent and fragment ions and not NMR data, this method remains important for the estimation of velvet antler composition and for providing reference values, particularly for the use of velvet antler in combination with various clinical drugs.

Velvet antler has been demonstrated to exhibit various anti-osteoporosis (29-31) anti-fatigue $(32,33)$, anti-inflammatory $(34,35)$ and anti-cancer $(36)$ effects, which are commonly associated with the chemical components of velvet antler. The identification velvet antler chemical components determined in the present study may facilitate further assessment into its bioactivity and functional mechanism.

The qualitative and quantitative detection of phospholipids in velvet antler was performed in the current study, which revealed that 16 phospholipids were present. The content of MPC in velvet antler was highest among the phospholipids and thus likely contributes to its biological effects, particularly that of anti-oxidation (37).

The phospholipids in velvet antler have been reported to have various biological actives, for example proliferation activity on spleen cells, and they are the subject of increasing research interest throughout the world (38). Herein, a 
systematic, sensitive bioanalytical UPLC/QTOF-MS/MS assay was developed to determine the content of phospholipids in velvet antler; this method will facilitate the quality control of velvet antler and can be widely applied in clinical settings.

The analysis of phospholipids in velvet antler using UPLC/QTOF-MS/MS has been demonstrated to be a suitable strategy for biomarker discovery $(18,21,23)$. A total of 16 phospholipids in the EVA samples were identified and three of these compounds were quantified. The current data revealed that the content of phospholipids was low in the EVA samples, hindering their detection by certain commonly used methods, including high performance liquid chromatography with UV detection. The method of UPLC/QTOF-MS/MS described in the current study adequately addressed this problem as this quantitative method exhibited great advantages in terms of ease of sample preparation, excellent recovery and high sensitivity. To the best of our knowledge, the lignans, alkaloids, organic acids, steroids and terpenoids identified in velvet antler were detected and tentatively characterized for the first time using the UNIFI platform. However, further pharmacological studies are required to explore the associations between velvet antler components and bioactivity. This may advance its application in clinical settings.

\section{Acknowledgements}

Not applicable.

\section{Funding}

The present study was supported by the Science and Technology Development Program of Jilin Province (grant nos. 201502040 37YY and 20160101072JC).

\section{Availability of data and materials}

All data generated or analyzed during the present study are included in this published article.

\section{Authors' contributions}

JHL designed all experiments, organized data and wrote this manuscript. LQZ determined the components of velvet antler using UPLC/QTOF/MS and analyzed the data. JW took off components from velvet antler with the method of ultrasound assisted extraction. TL screened the components of velvet antler with UNIFI platform and analyzed the data. PYL designed all experiments and performed data analysis. YHW took off components from velvet antler with the method of ultrasound assisted extraction and determined the components of velvet antler using UPLC/QTOF/MS. MY screened the components of velvet antler with UNIFI platform and analyzed the data. JPL designed experiments based on the references and analyzed the data.

\section{Ethics approval and consent to participate}

Not applicable.

\section{Patient consent for publication}

Not applicable.

\section{Competing interests}

The authors declare that they have no competing interests.

\section{References}

1. Price JS, Allen S, Faucheux C, Althnaian T and Mount JG: Deer antlers: A zoological curiosity or the key to understanding organ regeneration in mammals? J Anat 207: 603-618, 2005.

2. Clark DE, Li C, Wang W, Martin SK and Suttie JM: Vascular localization and proliferation in the growing tip of the deer antler. Anat Rec A Discov Mol Cell Evol Biol 288: 973-981, 2006.

3. Clark DE, Lord EA and Suttie JM: Expression of VEGF and pleiotrophin in deer antler. Anat Rec A Discov Mol Cell Evol Biol 288: 1281-1293, 2006.

4. Barling PM, Lai AK and Nicholson LF: Distribution of EGF and its receptor in growing red deer antler. Cell Biol Int 29: 229-236, 2005.

5. Lai AK, Hou WL, Verdon DJ, Nicholson LF and Barling PM: The distribution of the growth factors FGF-2 and VEGF, and their receptors, in growing red deer antler. Tissue Cell 39: 35-46, 2007.

6. Pita-Thomas W, Fernández-Martos C, Yunta M, Maza RM, Navarro-Ruiz R, Lopez-Rodríguez MJ, Reigada D, Nieto-Sampedro M and Nieto-Diaz M: Gene expression of axon growth promoting factors in the deer antler. PLoS One 5: e15706, 2010.

7. Jeon B, Kim S, Lee S, Park P, Sung S, Kim J and Moon S: Effect of antler growth period on the chemical composition of velvet antler in sika deer (Cervus nippon). Mamm Biol 74: 374-380, 2009.

8. Wang ZY, Shi SR, Shi YJ, Zhang J and Zhou QY: A comparison of methods to determine amino acid availability of feedstuffs in cecectomized ganders. Poult Sci 87: 96-100, 2008.

9. Pita-Thomas W, Nieto-Sampedro M, Maza RM and Nieto-Diaz M: Factors promoting neurite outgrowth during deer antler regeneration. J Neurosci Res 88: 3034-3047, 2010.

10. Zhou QL, Liu YQ, Wang Y, Guo YJ and Wang BX: A comparison of chemical composition and bioactivity of polypeptides from velvet antlers of Cervus nippon Temminck and Cervus elaphus Linnaeus. Zhongguo Zhong Yao Za Zhi 26: 699-702, 2001 (In Chinese).

11. Wang H, Lin Z, Liu Q, Cai MJ, Xu L and Zhang XZ: Preparation of velvet antlers small peptides and stimulating effects on osteosarcoma cell proliferation. Chem J Chinese Univ 29: 1791-1796, 2008.

12. Pita-Thomas W, Barroso-Garcia G, Moral V, Hackett AR, Cavalli $\mathrm{V}$ and Nieto-Diaz M: Identification of axon growth promoters in the secretome of the deer antler velvet. Neuroscience 340: 333-344, 2017.

13. Nieto-Diaz M, Pita-Thomas DW, Munoz-Galdeano T, Martinez-Maza C, Navarro-Ruiz R, Reigada D, Yunta M, Caballero-Lopez MJ, Nieto-Sampedro M and Martinez-Maza R: Deer antler innervation and regeneration. Front Biosci (Landmrk Ed) 17: 1389-1401, 2012.

14. Nieto-Diaz M, Pita-Thomas W, Maza RM, Yunta M, Lopez-Rodríguez MJ, Navarro-Ruiz R, Reigada D, Fernandez-Martos CM and Nieto-Sampedro M: Factors promoting axon growth in the deer antler. Anim Prod Sci 51: 351-354, 2011.

15. Wang H, Huang YB, Gao KX, Sun H and Gao ZL: Preparation and purification of velvet antlers peptides and its antioxidant activities. Chem J Chinese Univ 31: 2390-2395, 2010.

16. Lee SR, Jeon BT, Kim SJ, Kim MH, Lee SM and Moon SH: Effects of antler development stage on fatty acid, vitamin and GAGS contents of velvet antler in spotted deer (Cervus nippon). Asian Austral J Anim 20: 1546-1550, 2007.

17. Zhao M, Tao JH, Du LY, Jiang S, Qian DW and Duan JN: UPLC-Q-TOF/MS-based metabolic profiling comparison of two major bioactive components and Their metabolites in normal and CKD rat plasma, urine and feces following oral administration of fructus corni extract. J Chromatogr Sci 55: 857-865, 2017. 
18. Tao JH, Zhao M, Wang DG, Yang C, Chen GT, Zhao X, Pu XL and Jiang S: UPLC-Q-TOF/MS-based screening and identification of two major bioactive components and their metabolites in normal and CKD rat plasma, urine and feces after oral administration of Rehmannia glutinosa Libosch extract. J Chromatogr B Analyt Technol Biomed Life Sci 1001: 98-106, 2015.

19. Dong YF, Tang MH, Song H, Li R, Wang C, Ye H, Qiu N, Zhang Y, Chen $L$ and Wei Y: Characterization of metabolic profile of honokiol in rat feces using liquid chromatography coupled with quadrupole time-of-flight tandem mass spectrometry and (13)C stable isotope labeling. J Chromatogr B Analyt Technol Biomed Life Sci 953-954: 20-29, 2014.

20. Liu J, Tang MH, Lai HJ, Dong Y, Xie C, Ye H, Ma L, Qiu N, Li Y, Cai L and Chen L: Identification of metabolites of honokio in rat urine using $13 \mathrm{C}$ stable isotope labeling and liquid chromatography coupled with quadrupole time-of-flight tandem mass spectrometry. J Chromatogr A 1295: 48-56, 2013.

21. Taguchi R, Houjou T, Nakanishi H, Yamazaki T, Ishida M, Imagawa $\mathrm{M}$ and Shimizu T: Focused lipidomics by tandem mass spectrometry. J Chromatogr B Analyt Technol Biomed Life Sci 823: 26-36, 2005.

22. Skwarek LC and Boulianne GL: Great expectations for PIP: Phosphoinositides as regulators of signaling during development and disease. Dev Cell 16: 12-20, 2009.

23. Zitouni M, Wewer V, Dörmann P, Abdelly C and Ben Youssef N Quadrupole time-of-flight mass spectrometry analysis of glycerophospholipid molecular species in the two halophyte seed oils: Eryngium maritimum and Cakile maritima. Food Chem 213: 319-328, 2016

24. Ejsing CS, Sampaio JL, Surendranath V, Duchoslav E, Ekroos K, Klemm RW, Simons K and Shevchenko A: Global analysis of the yeast lipidome by quantitative shotgun mass spectrometry. Proc Natl Acad Sci USA 106: 2136-2141, 2009.

25. Ejsing CS, Bilgin M and Fabregat A: Quantitative profiling of long-chain bases by mass tagging and parallel reaction monitoring. PLoS One 10: e0144817, 2015.

26. Zhou R and Li SF: Supercritical carbon dioxide and co-scosolvent extractions of estradiol and progesterone from antler velvet. J Food Compos Anal 22: 72-78, 2009.

27. Zhang FX, Li M, Qiao LR, Yao ZH, Li C, Shen XY, Wang Y, Yu K, Yao XS and Dai Y: Rapid characterization of Ziziphi Spinosae Semen by UPLC/Qtof MS with novel informatics platform and its application in evaluation of two seeds from Ziziphus species. J Pharm Biomed Anal 122: 59-80, 2016.
28. Qiu S, Yang WZ, Yao CL, Qiu ZD, Shi XJ, Zhang JX, Hou JJ, Wang QR, Wu WY and Guo DA: Nontargeted metabolomic analysis and 'commercial-homophyletic' comparison-induced biomarkers verification for the systematic chemical differentiation of five different parts of Panax ginseng. J Chromatogr A 1453: 78-87, 2016

29. LiZH,Zhao WH and Zhou QL: Experimental study of velvet antler polypeptides against oxidative damage of osteoarthritis cartilage cells. Zhongguo Gu Shang 24: 245-248, 2011 (In Chinese).

30. Zhang Z, Liu X, Duan L, Li X, Zhang Y and Zhou Q: The effects of velvet antler polypeptides on the phenotype and related biological indicators of osteoarthritic rabbit chondrocytes. Acta Biochim Pol 58: 297-302, 2011.

31. Li YJ, Kim TH, Kwak HB, Lee ZH, Lee SY and Jhon GJ: Chloroform extract of deer antler inhibits osteoclast differentiation and bone resorption. J Ethnopharmacol 113: 191-198, 2007.

32. Sui Z, Zhang L, Huo Y and Zhang Y: Bioactive components of velvet antlers and their pharmacological properties. J Pharmaceut Biomed 87: 229-240, 2014.

33. Wu FF, Li HQ, Jin LJ, Li X, Ma Y, You J, Li S and Xu Y: Deer antler base as a traditional Chinese medicine: A review of its traditional uses, chemistry and pharmacology. J Ethnopharmacol 145: 403-415, 2013.

34. Suh SJ, Kim KS, Lee AR, Ha KT, Kim JK, Kim DS, Lee YC, Kim MS, Kwon DY and Kim CH: Prevention of collagen-induced arthritis in mice by Cervus korean TEMMINCK var. mantchuricus Swinhoe. Environ Toxicol Phar 23: 147-153, 2007.

35. Kim KS, Choi YH, Kim KH, Lee YC, Kim CH, Moon SH, Kang SG and Park YG: Protective and anti-arthritic effects of deer antler aqua-acupuncture (DAA), inhibiting dihydroorotate dehydrogenase, on phosphate ions-mediated chondrocyte apoptosis and rat collagen-induced arthritis. Int Immunopharmacol 4: 963-973, 2004.

36. Fraser A, Haines SR, Stuart EC, Scandlyn MJ, Alexander A, Somers-Edgar TJ and Rosengren RJ: Deer velvet supplementation decreases the grade and metastasis of azoxymethane-induced colon cancer in the male rat. Food Chem Toxicol 48: 1288-1292, 2010.

37. Zhou R and Li SF: In vitro antioxidant analysis and characterisation of antler velvet extract. Food Chem 114: 1321-1327, 2009.

38. Landete Castillejos T, Estevez JA, Martinez A, Ceacero F, Garcia A and Gallego L: Does chemical composition of antler bone reflect the physiological effort made to grow it. Bone 40: 1095-1102, 2007. 